Araştırma Makalesi - Research Article

\title{
Kuantum Tam Diferansiyel ve Uygulaması
}

\author{
Zülal Mısır1* ${ }^{*}$ Ömer Faruk Gözükızı1 ${ }^{2}$
}

\section{öZ}

Kabul / Accepted: 06/03/2020

$\mathrm{Bu}$ çalışmada temel kuantum analiz bilgileri kullanılarak, kuantum diferansiyel denklemini tam yapan koşullar ile klasik diferansiyel denklemini tam yapan koşulların aynı olduğu gösterilmiştir. Ardından anlatılanlarla ilgili bir örnek verilmiştir. 


\title{
Quantum Exact Differential and Application
}

\begin{abstract}
In this article, we show that the conditions that make the exact quantum differential equation the same as with that make the exact ordinary differential equation using basic quantum calculus. Then, an example was given of what was described.
\end{abstract}

Keywords-Quantum calculus, Exact differential equation, Quantum exact differential equation 


\section{I.GİRIŞ}

Limitsiz analiz adı verilen kuantum analiz, limit kavramı olmadan geleneksel sonsuz küçük hesaplara eşdeğerdir. Kuantum analiz, q, kuantum kelimesini temsil etmek üzere, ‘q-analiz' şeklinde ifade edilir [1].

Bu makalede öncelikle kuantum analizdeki q-diferansiyel;

$$
d_{q} f(x)=f(q x)-f(x)
$$

ve q-türev;

$$
D_{\mathrm{q}} f(x)=\frac{d_{q} f(x)}{d_{q^{x}}}=\frac{f(q x)-f(x)}{(q-1) x}
$$

ifadeleri tanımlanmıştır. Burada $0<q<1$ olarak alınmıştır [2]. Bu tanımlamalar, iki değişkenli fonksiyona uygulanarak, iki değişkenli fonksiyon için kuantum kısmi türev tanımlamaları da yapılmıştır.

Ardından makalede kullanılacak olan Jackson integralini tanımlayabilmek için, ihtiyaç duyulan qantitürev kavramı kısaca açıklanarak, Jackson Integral;

$$
\int f(x) d_{q} x=(1-q) x \sum_{j=0}^{\infty} q^{j} f\left(q^{j} x\right)
$$

şeklinde tanımlanmıştır [3].

$\mathrm{Bu}$ tanımlamaların ardından makalenin asıl konusu olan kuantum diferansiyel denkleminin tamlık koşulunun, klasik analizdeki diferansiyel denklemin tamlık koşulu ile aynı olduğu gösterilmiştir. Son olarak bir örnek ile açıklanmıştır [4].

\section{II. q-DİFERANSIYEL ve q-TÜREV}

\section{A. q-diferansiyel}

Verilen bir $f(x)$ fonksiyonunun q-diferansiyeli (4) eşitliğiyle tanımlanmaktadır:

$$
d_{q} f(x)=f(q x)-f(x)
$$

ayrıca q-türev eşitliğinde kullanılacak olan $d_{q} x$ ifadesi aşağıdaki şekilde tanımlanmaktadır:

$$
d_{q} x=(q-1) x
$$

B. q-türev

1) Tanım (Tek değişkenli fonksiyon için q-türev): Kuantum diferansiyel ile uyumlu olan kuantum türev;

$$
D_{q} f(x)=\frac{d_{q} f(x)}{d_{q^{x}}}=\frac{f(q x)-f(x)}{(q-1) x}
$$

şeklindedir.

2) Tanım (ìki değişsenli fonksiyon için q-türev):

$$
\begin{aligned}
& D_{q_{x}} f(x, y)=\frac{d_{q} f(x, y)}{d_{q} x}=\frac{f(q x, y)-f(x, y)}{(q-1) x} \\
& D_{q_{y}} f(x, y)=\frac{d_{q} f(x, y)}{d_{q} y}=\frac{f(x, q y)-f(x, y)}{(q-1) y}
\end{aligned}
$$


şeklindedir.

\section{III. q-ANTITÜREV}

\section{A. Tanim (q-antitürev)}

Eğer, $D_{q} F(x)=f(x)$ ise, $F(x)$ fonksiyonu $f(x)$ fonksiyonunun q-antitürevidir. Bir fonksiyonun $q-$ antitürevi aşağıdaki gibi tanımlanır;

$$
F(x)=\int \mathrm{f}(\mathrm{x}) \mathrm{d}_{\mathrm{q}} \mathrm{x}
$$

Klasik analizde antitürev tek değildir. Türev alınırken sabit ifadeler yok olduğu için, antitürev içinde sonsuz sayıda farklı sabit ifade olabilir. Bu durum farklı antitürevler bulunmasını sağlar ve teklik durumu ortadan kalkar.

Kuantum analizdeki durum daha güç algılanır. q-analizde sadece $\varphi(q x)=\varphi(x)$ olduğu durumda $\mathrm{D}_{\mathrm{q}} \varphi(\mathrm{x})=0$ olur. Bu gibi bir $\varphi$ fonksiyonuna herhangi bir sabit eklenmesi, bu fonksiyonun q-türevini değiştirmez. Ancak bununla birlikte, biz $\varphi$ 'nin kuvvet serisi olmasına ihtiyaç duyarsak; $\varphi(\mathrm{qx})=\varphi(\mathrm{x})$ durumu her bir $\mathrm{n}$ için, $\mathrm{q}^{\mathrm{n}} \mathrm{c}_{\mathrm{n}}=\mathrm{c}_{\mathrm{n}}$ eşitliğini işaret eder. ( $\mathrm{c}_{\mathrm{n}}$ burada $\mathrm{x}^{\mathrm{n}}$ 'nin katsayısıdır.) Bu durum sadece, $\mathrm{n} \geq 1$ için $\mathrm{c}_{\mathrm{n}}=$ 0 olduğu zaman mümkündür, yani $\varphi$ sabittir. Bu yüzden, eğer;

$$
f(x)=\sum_{n=0}^{\infty} \alpha_{n} x^{n}
$$

şeklinde tanımlanan ifade bir kuvvet serisi ise, $\mathrm{f}(\mathrm{x})$ bir sabit terim eklenene kadar;

$$
\int f(x) d_{q} x=\sum_{n=0}^{\infty} \frac{\alpha_{n} x^{n+1}}{[n+1]}+C
$$

şeklinde olan tek bir q-antitüreve sahiptir [3].

\section{JACKSON INTEGRAL}

\section{A. Tanim}

$\hat{x}$ ve $\widehat{M}_{q}$ polinom uzayı üzerinde lineer operatörler olsunlar. Bu operatörlerin $f(x)$ fonksiyonu üzerine işlemleri aşağıdaki eşitliklerle gösterilmiştir:

$$
\begin{aligned}
& \hat{x}[f(x)]=x f(x) \\
& \widehat{M}_{q}[f(x)]=f(q x)
\end{aligned}
$$

\section{B. Tanım}

$f(x)$ keyfi şekilde tanımlanan bir fonksiyon olsun. Bu fonksiyonun q-antitürevi olan $F(x)$ 'i bulmak için $\widehat{\mathrm{M}}_{\mathrm{q}}$ operatörü kullanılacaktır. Öyleyse, q-türevin tanımından;

$$
\frac{1}{(\mathrm{q}-1) \mathrm{x}}\left(\widehat{\mathrm{M}}_{\mathrm{q}}-1\right) \mathrm{F}(\mathrm{x})=\frac{\mathrm{F}(\mathrm{qx})-\mathrm{F}(\mathrm{x})}{(\mathrm{q}-1) \mathrm{x}}=\mathrm{f}(\mathrm{x})
$$

eşitliğinin var olduğu açıktır.

Geometrik seri makalede kullanılacak olup, aşağıda verilmiştir:

$$
\mathrm{s}=1+\mathrm{r}+\mathrm{r}^{2}+\mathrm{r}^{3}+\cdots=\sum_{\mathrm{k}=0}^{\infty} \mathrm{r}^{\mathrm{k}}=\frac{1}{1-\mathrm{r}} \quad(0<\mathrm{r}<1)
$$


Sıranın önemi olduğu vurgulanmalıdır, çünkü operatörler değişmez. q-antitürev; geometrik seri ifadesi kullanılarak aşağıdaki gibi formüle edilip yazılabilir:

$$
\mathrm{F}(\mathrm{x})=\frac{1}{1-\widehat{\mathrm{M}}_{\mathrm{q}}}=((1-\mathrm{q}) \mathrm{xf}(\mathrm{x}))=(1-\mathrm{q}) \sum_{\mathrm{j}=0}^{\infty} \widehat{\mathrm{M}}_{\mathrm{q}}{ }^{\mathrm{j}}(\mathrm{xf}(\mathrm{x}))
$$

Böylece;

$$
\int f(x) d_{q} x=(1-q) x \sum_{j=0}^{\infty} q^{j} f\left(q^{j} x\right)
$$

elde edilir. Bu seri f(x)'in Jackson integrali olarak adlandırılmaktadır [3].

\section{V. İKİ DEĞİSSENLİ FONKSIYON IÇCiN q-TAM DİFERANSIYEL DENKLEM}

\section{A. Tanım (q-Tam diferansiyel denklem)}

$$
P(x, y) d_{q} x+Q(x, y) d_{q} y=0
$$

q-diferansiyel denklemi bir $\mathrm{f}(\mathrm{x}, \mathrm{y})=\mathrm{c}$ fonksiyonunun $\mathrm{q}$-tam diferansiyelini ifade ediyorsa bu denkleme q-tam diferansiyel denklem denir. $\mathrm{O}$ halde $\mathrm{f}$ fonksiyonunun $\mathrm{q}$-tam diferansiyeli:

$$
\begin{aligned}
& \mathrm{d}_{\mathrm{q}} \mathrm{f}(\mathrm{x}, \mathrm{y})=\mathrm{P}(\mathrm{x}, \mathrm{y}) \mathrm{d}_{\mathrm{q}} \mathrm{x}+\mathrm{Q}(\mathrm{x}, \mathrm{y}) \mathrm{d}_{\mathrm{q}} \mathrm{y} \\
& \mathrm{d}_{\mathrm{q}} f(x, y)=\frac{d_{q} f}{d_{q} x} d_{q} x+\frac{d_{q} f}{d_{q} y} d_{q} y
\end{aligned}
$$

şeklinde olmalıdır. Buradan;

$$
\begin{aligned}
& P(x, y)=\frac{d_{q} f}{d_{q^{x}}} \\
& Q(x, y)=\frac{d_{q^{f}}}{d_{q} y}
\end{aligned}
$$

şartları ortaya çıkar. Bu şartlardan da;

$$
\begin{aligned}
& P_{q_{y}}(x, y)=\frac{d_{q}{ }^{2} f}{d_{q^{x}} d_{q y}} \\
& Q_{q_{x}}(x, y)=\frac{d_{q^{2}}{ }^{2}}{d_{q} y d_{q} x}
\end{aligned}
$$

olur. q-tam diferansiyel olması için;

$$
\frac{d_{q}^{2} f}{d_{q} x d_{q} y}=\frac{d_{q}{ }^{2} f}{d_{q} y d_{q} x}
$$

yani;

$$
P_{\mathrm{q}_{\mathrm{y}}}(\mathrm{x}, \mathrm{y})=\mathrm{Q}_{\mathrm{q}_{\mathrm{x}}}(\mathrm{x}, \mathrm{y})
$$

eşitliğinin sağlanması gerekir.

Şimdi q-diferansiyel denklem için bu eşitliğin sağlandığını gösterelim: 


$$
\begin{aligned}
& \frac{d_{q} f(x, y)}{d_{q} x}=D_{q_{x}} f(x, y)=\frac{f(q x, y)-f(x, y)}{(q-1) x} \\
& \frac{d_{q}^{2} f(x, y)}{d_{q} x d_{q y} y}=D_{q_{x y}} f(x, y)=\frac{\frac{f(q x, q y)-f(x, y y)}{(q-1) x}-\left[\frac{f(q x, y)-f(x, y)}{(q-1) x}\right]}{(q-1) y} \\
& \frac{d_{q}^{2} f(x, y)}{d_{q} x d_{q} y}=\frac{f(q x, q y)-f(x, q y)-f(q x, y)+f(x, y)}{(q-1)^{2} x y} \\
& \frac{d_{q} f(x, y)}{d_{q} y}=D_{q_{y}} f(x, y)=\frac{f(x, q y)-f(x, y)}{(q-1) y} \\
& \frac{d_{q}{ }^{2} f(x, y)}{d_{q} y d_{q} x}=D_{q_{y x}} f(x, y)=\frac{\frac{f(q x, q y)-f(q x, y)}{(q-1) y}-\left[\frac{f(x, q y)-f(x, y)}{(q-1) y}\right]}{(q-1) x} \\
& \frac{d_{q}^{2} f(x, y)}{d_{q} y d_{q} x}=\frac{f(q x, q y)-f(x, q y)-f(q x, y)+f(x, y)}{(q-1)^{2} x y}
\end{aligned}
$$

olarak elde edilir. Buradan, (29) ve (32) eşitliklerinin eşit olduğu görülmektedir. Yani bir q-diferansiyel denklemin tam diferansiyel denklem olabilmesi için (25) yani (26) eşitliğinin sağlanıyor olması gerekir.

\section{VI. ÖRNEK}

$$
[x y(q+1)+1] d_{q} x+\left[x^{2}\right] d_{q} y=0
$$

q-diferansiyel denklemi, q-tam diferansiyel denklem midir? Eğer öyle ise genel çözümü elde ediniz. Burada q, $0<\mathrm{q}<1$ aralığındadır.

\section{A. Çözüm}

Bu denklemde;

$$
\begin{aligned}
& P(x, y)=x y(q+1)+1 \\
& Q(x, y)=x^{2}
\end{aligned}
$$

dir. Bu eşitlikleri kullanarak (26) eşitliğinin sağlanıp sağlanmadığını kontrol edilmelidir. (7)-(8) ile gösterilmiş olan q-kısmi türev bağıntılarından faydalanarak;

$$
\begin{aligned}
P_{q_{y}}(x, y) & =\frac{P(x, q y)-P(x, y)}{(q-1) y} \\
& =\frac{x q y(q+1)+1-(x y(q+1)+1)}{(q-1) y} \\
& =\frac{q^{2} x y+x q y+1-x q y-x y-1}{(q-1) y} \\
& =\frac{q^{2} x y-x y}{(q-1) y} \\
& =\frac{x y[(q-1)(q+1)]}{(q-1) y} \\
& =x(q+1)
\end{aligned}
$$

şeklinde elde edilir. Ardından; 


$$
\begin{aligned}
Q_{\mathrm{q}_{\mathrm{x}}}(\mathrm{x}, \mathrm{y}) & =\frac{\mathrm{Q}(\mathrm{qx}, \mathrm{y})-\mathrm{Q}(\mathrm{x}, \mathrm{y})}{(\mathrm{q}-1) \mathrm{x}} \\
& =\frac{\mathrm{q}^{2} \mathrm{x}^{2}-\mathrm{x}^{2}}{(\mathrm{q}-1) \mathrm{x}} \\
& =\frac{\mathrm{x}^{2}[(\mathrm{q}-1)(\mathrm{q}+1)]}{(\mathrm{q}-1) \mathrm{x}} \\
& =x(\mathrm{q}+1)
\end{aligned}
$$

olarak elde edilir. Buradan;

$$
\begin{aligned}
& P_{q_{y}}(x, y)=x(q+1)=x(q+1)=Q_{q_{x}}(x, y) \\
& P_{q_{y}}(x, y)=Q_{q_{x}}(x, y)
\end{aligned}
$$

olarak bulunarak, verilen denklem q-tam diferansiyel denklem olma koşulunu sağlamaktadır.

Şimdi denklemin genel çözümü elde edilecektir.

$$
P(x, y)=\frac{d_{q} f}{d_{q x}}
$$

olduğu ifade edilmişti. Buradan yola çıkarak $\mathrm{f}(\mathrm{x}, \mathrm{y})=\mathrm{c}$ türünde, verilen denklemin çözümü olan bir fonksiyon araştırılacaktır.

$$
d_{q} f(x, y)=P(x, y) d_{q} x
$$

Bu eşitlikte her iki tarafın x'e göre q-integrali alınırsa;

$$
\begin{aligned}
& \int d_{q} f(x, y)=\int P(x, y) d_{q} x=c \\
& f(x, y)=\int[x y(q+1)+1] d_{q} x=c
\end{aligned}
$$

bu hale geldikten sonra, (17) ile belirtilen Jackson integral tanımından, (43) ile verilen eşitliğin sağ tarafı aşağıdaki gibi yazılabilir:

$$
\begin{aligned}
& f(x, y)=(1-q) x \sum_{j=0}^{\infty} q^{j}\left(q^{j} x y(q+1)+1\right)=c \\
& f(x, y)=(1-q) x \sum_{j=0}^{\infty} q^{j}\left(q^{j+1} x y+q^{j} x y+1\right)=c \\
& f(x, y)=(1-q) x \sum_{j=0}^{\infty}\left(q^{2 j+1} x y+q^{2 j} x y+q^{j}\right)=c \\
& f(x, y)=(1-q) x\left(\sum_{j=0}^{\infty} q^{2 j+1} x y+\sum_{j=0}^{\infty} q^{2 j} x y+\sum_{j=0}^{\infty} q^{j}\right)=c \\
& f(x, y)=(1-q) x\left(\begin{array}{c}
q x y+q^{3} x y+q^{5} x y+\cdots+q^{2 j+1} x y+\cdots \\
+q^{0} x y+q^{2} x y+q^{4} x y+\cdots+q^{2 j} x y+\cdots \\
+q^{0}+q^{1}+q^{2}+\cdots+q^{j}+\cdots
\end{array}\right)=c \\
& f(x, y)=(1-q) x\left(\begin{array}{c}
x y\left\{q^{0}+q^{1}+q^{2}+\cdots+q^{2 j+1}\right\}+\cdots \\
+\left\{q^{0}+q^{1}+q^{2}+\cdots+q^{j}\right\}+\cdots
\end{array}\right)=c
\end{aligned}
$$


$0<\mathrm{q}<1$ olduğu için, (15) eşitliğinden faydalanarak;

$$
\begin{aligned}
& f(x, y)=(1-q) x\left(x y \frac{1}{1-q}+\frac{1}{1-q}\right)=c \\
& f(x, y)=(1-q) x\left(\frac{x y+1}{1-q}\right)=c \\
& f(x, y)=x(x y+1)=c \\
& f(x, y)=x^{2} y+x+c(y)=0
\end{aligned}
$$

elde edilerek ilk aşama tamamlanmış olur. Şimdi;

$$
Q(x, y)=\frac{d_{q} f}{d_{q} y}
$$

eşitliğinden faydalanarak ve

$$
f(x, y)=x^{2} y+x+c(y)=0
$$

eşitliği kullanılarak;

$$
\begin{aligned}
& \frac{x^{2} q y+x-x^{2} y-x}{(q-1) y}+\frac{d_{q} c(y)}{d_{q} y}=x^{2} \\
& \frac{x^{2} y(q-1)}{(q-1) y}+\frac{d_{q} c(y)}{d_{q} y}=x^{2} \\
& x^{2}+\frac{d_{q} c(y)}{d_{q} y}=x^{2} \\
& \frac{d_{q} c(y)}{d_{q} y}=0 \\
& d_{q} c(y)=0
\end{aligned}
$$

Her iki tarafın y-e göre q-integrali alınırsa;

$$
\int \mathrm{d}_{\mathrm{q}} \mathrm{c}(\mathrm{y})=\int 0
$$

olur ve buradan;

$$
c(y)=C
$$

elde edilir. Böylelikle verilen denklemin çözüm fonksiyonu:

$$
f(x, y)=x^{2} y+x+C
$$

olarak elde edilmiş olur. 


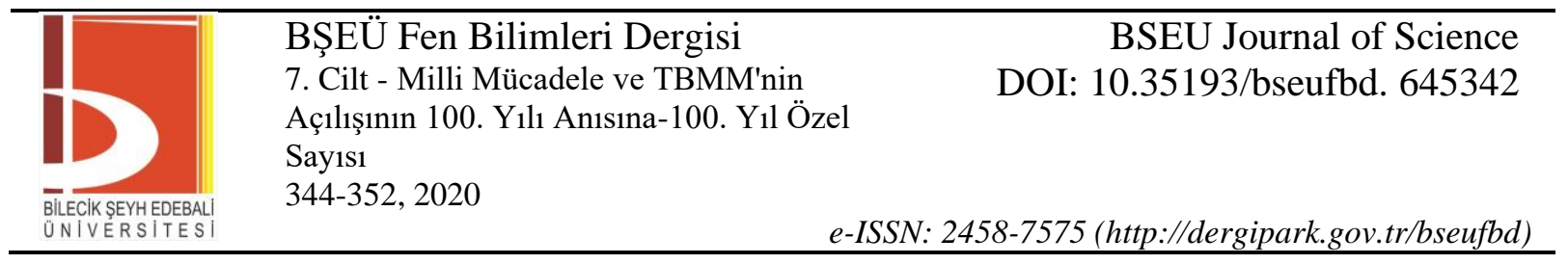

\section{KAYNAKLAR}

[1] https://en.wikipedia.org/wiki/Quantum_calculus. Erişim Tarihi: 26.02.2019

[2] Ciavarella, A. 2016. What Is q-Calculus? Course Hero, 1-6 pages.

[3] Kac, V., Cheung, P. 2002. Quantum Calculus. 1.Bask1, Springer-Verlag, New York.

[4] Misır, Z. 2019. Kuantum Analiz ve Uygulamaları. Y.Lisans Tezi, Sakarya Üniversitesi, Fen Bilimleri Enstitüsü, Matematik Bölümü 